\title{
Diagnostic ability of contrast-enhanced computed tomography for metastatic cervical nodes in head and neck squamous cell carcinomas: significance of additional coronal reconstruction images
}

\author{
Tomohiro Ando ${ }^{A, B, D, E, F}$, Hiroki Kato ${ }^{A, B, C, D, E}$, Masaya Kawaguchi ${ }^{B}$, Yukichi Tanahashi ${ }^{B, D}$, Mitsuhiro Aoki ${ }^{B}$, \\ Bunya Kuze ${ }^{B}$, Masayuki Matsuo ${ }^{D, E}$
}

Gifu University School of Medicine, Japan

\section{Abstract}

\begin{abstract}
Purpose: To evaluate the significance of additional coronal reconstruction images in the diagnostic ability of contrastenhanced computed tomography (CECT) for metastatic cervical nodes in patients with head and neck squamous cell carcinomas (HNSCC).

Material and methods: We retrospectively assessed 97 metastatic and 141 reactive histologically proven cervical nodes of 38 patients with HNSCC, who underwent CECT before neck dissection. Observer \#1, an expert radiologist in head and neck imaging, and observer \#2, a general radiologist, reviewed all CECT images. The observers first assessed the presence of nodal metastasis using axial CECT alone (A-CECT). Three days later, they reassessed its presence using combined axial and coronal CECT (A\&C-CECT).
\end{abstract}

Results: The sensitivity of A-CECT vs. A\&C-CECT was $73.2 \%$ vs. $75.3 \%$ for observer \#1 ( $p=0.73$ ) and $69.1 \%$ vs. $69.1 \%$ for observer \#2 ( $p=1.00$ ), respectively. The specificity of A-CECT versus A\&C-CECT was $92.2 \%$ vs. 97.2\% for observer \#1 ( $p<0.05)$ and $92.9 \%$ vs. $95.7 \%$ for observer \#2 ( $p=0.22)$, respectively. The accuracy of A-CECT versus A\&C-CECT was $84.5 \%$ vs. $88.2 \%$ for observer $\# 1$ ( $p<0.05)$ and $83.2 \%$ vs. $85.3 \%$ for observer $\# 2(p=0.30)$, respectively. The area under the curve (AUC) of A-CECT vs. A\&C-CECT was 0.86 vs. 0.91 for observer \#1 $(p<0.05)$ and 0.85 vs. 0.85 for observer \#2 ( $p=0.80)$, respectively.

Conclusions: The specificity, accuracy, and AUC increased with the use of coronal images during the assessment by the expert radiologist. The appropriate use of coronal images allowed proper configuration recognition and improved diagnostic ability.

Key words: head and neck squamous cell carcinomas, lymph node metastasis, CT, coronal reconstruction images.

\section{Introduction}

The presence of metastatic cervical nodes is one of the most significant prognostic factors in patients with head and neck squamous cell carcinoma (HNSCC) because it influences the therapeutic strategy and clinical outcome [1-5]. Palpation alone is often insufficient for the proper evaluation of cervical nodes, and radiographic imaging is useful for the assessment of anatomically deep cervical nodes, including retropharyngeal nodes [6,7]. Contrast-enhanced computed tomography (CECT) and magnetic resonance imaging (MRI) are widely used for the evaluation of nodal metastasis in HNSCC, and the size criterion of a maximum short-axis diameter of $10 \mathrm{~mm}$, as proposed by van den Brekel et al. [8], has gained widespread acceptance. Central necrosis and indistinct margins indicating extranodal spread are reliable morphological features for the diagnosis of malignancy, particularly in small $(<10 \mathrm{~mm})$ nodes $[9,10]$. Loss of fatty hilum is also a morphological feature suggestive of malignancy. However, although rela-

Correspondence address:

Hiroki Kato, Gifu University School of Medicine, Japan, e-mail: hkato@gifu-u.ac.jp

Authors' contribution:

A Study design · B Data collection · C Statistical analysis · D Data interpretation · E Manuscript preparation · F Literature search · G Funds collection 
tively high specificity of these morphological criteria has been reported, the sensitivity based on the size criteria on CT (71\%) [8] and loss of fatty hilum on ultrasonography (30\%) [11] is insufficient for the detection of nodal metastases.

A meta-analysis of 16 studies regarding the diagnostic ability of CT and MRI in detecting metastatic cervical nodes in HNSCC found that the sensitivity was $64.2 \%$ and $67.4 \%$ and the specificity was $75.4 \%$ and $78.7 \%$ [12] for CT and MRI, respectively. In contrast, a meta-analysis of 13 studies with per-neck-level data found that the sensitivity was higher for ${ }^{18} \mathrm{~F}$-fluorodeoxyglucose $\left({ }^{18} \mathrm{~F}-\mathrm{FDG}\right)$ PET/CT (84\%) than CT, MRI, and CT/MRI (63\%) [13]. However, because ${ }^{18} \mathrm{~F}-\mathrm{FDG}$-PET/CT is expensive and not easily available, CT and MRI are widely performed in patients with HNSCC. Therefore, it has been expected that CT and MRI show higher diagnostic performance for the detection of metastatic cervical nodes in HNSCC.

Table 1. Patient characteristics

\begin{tabular}{|l|c|}
\hline Parameter & 38 \\
\hline Number of patients, $N$ & \\
\hline Age (year) & 66.1 \\
\hline Mean & $32-88$ \\
\hline Range & \\
\hline Gender, $n$ & 29 \\
\hline Male & 9 \\
\hline Female & 9 \\
\hline Primay tumor site, $n$ & 9 \\
\hline Tongue & 6 \\
\hline Gingiva & 4 \\
\hline Hypopharynx & 3 \\
\hline Larynx & 3 \\
\hline Oropharynx & 21 \\
\hline Skin & 24 \\
\hline Palate & 10 \\
\hline Maxillary sinus & 4 \\
\hline Nasal cavity & 1 \\
\hline Histological tumor grade, $n$ & \\
\hline Well-differentiated & \\
\hline Moderately-differentiated & \\
\hline Poorly-differentiated & \\
\hline UICC clinical stage, $n$ & \\
\hline I & \\
\hline III & \\
\hline IV & \\
\hline
\end{tabular}

UICC - Union for International Cancer Control
With the widespread use of multi-detector computed tomography (MDCT), multiplanar reformation images are easily available in daily clinical practice. In the head and neck region, coronal reconstruction images are useful for identifying anatomical locations and evaluating morphological features. By adding the evaluation of cervical nodes using coronal images, the configuration recognition is often modified, in contrast to the evaluation using axial images alone. Although a few studies have compared the diagnostic ability between axial and coronal images [14-16], to the best of our knowledge, the utility of coronal images in addition to axial images for the diagnosis of metastatic cervical nodes in HNSCC has not been reported. Thus, the purpose of this study was to evaluate the significance of additional coronal reconstruction images in the diagnostic ability of CECT to identify metastatic cervical nodes in HNSCC.

\section{Material and methods}

\section{Patients}

The study was approved by the Research Ethics Committee of our Institutional Review Board and complied with the guidelines of the Health Insurance Portability and Accountability Act. The requirement for written, informed consent was waived due to the retrospective nature of this study. Between January 2015 and February 2018, the electronic medical records at our University Hospital were searched for the details of patients with HNSCC, who had undergone CECT within two months before neck dissection. After excluding patients who underwent chemotherapy or radiotherapy between CECT and surgery, 38 consecutive patients (29 males and 9 females; mean age, 66 years; age range, 32-88 years) were identified. The primary tumour sites were tongue $(n=9)$, gingiva $(n=9)$, hypopharynx $(n=6)$, larynx $(n=4)$, oropharynx $(n=3)$, skin $(n=3)$, palate $(n=2)$, maxillary sinus $(n=1)$, and nasal cavity $(n=1)$. The histological tumour grades of HNSCC were well differentiated in 24 patients, moderately differentiated in 10 , and poorly differentiated in four. The clinical tumour stages according to the Union for International Cancer Control classification were stage I in two patients, stage II in seven, stage III in eight, and stage IV in 21 . The patient characteristics are summarised in Table 1.

\section{Computed tomography imaging}

CT imaging was performed using a 64-slice CT system (Brilliance 64; Philips, Best, The Netherlands or Discovery 750 HD; GE Healthcare, Milwaukee, WI, USA). Axial and coronal CECT (A\&C-CECT) images were obtained for all patients and reconstructed at a section thickness of $2.5-\mathrm{mm}$ with no overlap. Single-phase CECT imaging was started 45 seconds after initiating an intravenous bolus injection of $100 \mathrm{ml}$ of nonionic iodine contrast material (Optiray 240 
[240 mg of iodine per ml]; Mallinckrodt Inc., Hazelwood, $\mathrm{MO}, \mathrm{USA}$ ) at an injection rate of $2 \mathrm{ml}$ per second.

\section{Image assessment}

An expert radiologist with 19 years of post-training experience in head and neck imaging (observer \#1) and a general radiologist with six years of post-training experience (observer \#2) independently reviewed all CECT images. Both the observers were blinded to the patient data. They assessed cervical nodes on CECT images without observing the primary tumours to minimise the diagnostic bias for nodal metastasis. They first assessed the presence of nodal metastasis using axial CECT (A-CECT) alone. Three days later, they reassessed the presence of nodal metastasis using combined axial and coronal CECT (A\&CCECT) in a randomised fashion. The observers evaluated the presence or absence of nodal metastasis according to the following criteria. Lymph nodes were confirmed as positive for metastasis if they were $\geq 10 \mathrm{~mm}$ in maximum short-axis diameter, contained central necrosis, or had indistinct margins. If the above-mentioned positive findings were not observed, the loss of fatty hilum or spherical (round)-shaped configuration was considered an ancillary metastatic feature. A confidence level for the presence of metastatic nodes was assigned for each lymph node according to a four-point scale: 1 , definitely absent; 2 , probably absent; 3 , probably present; and 4 , definitely present.

\section{Lymph node assessment}

The location of cervical nodes was divided into seven different levels in the imaging-based nodal classification reported

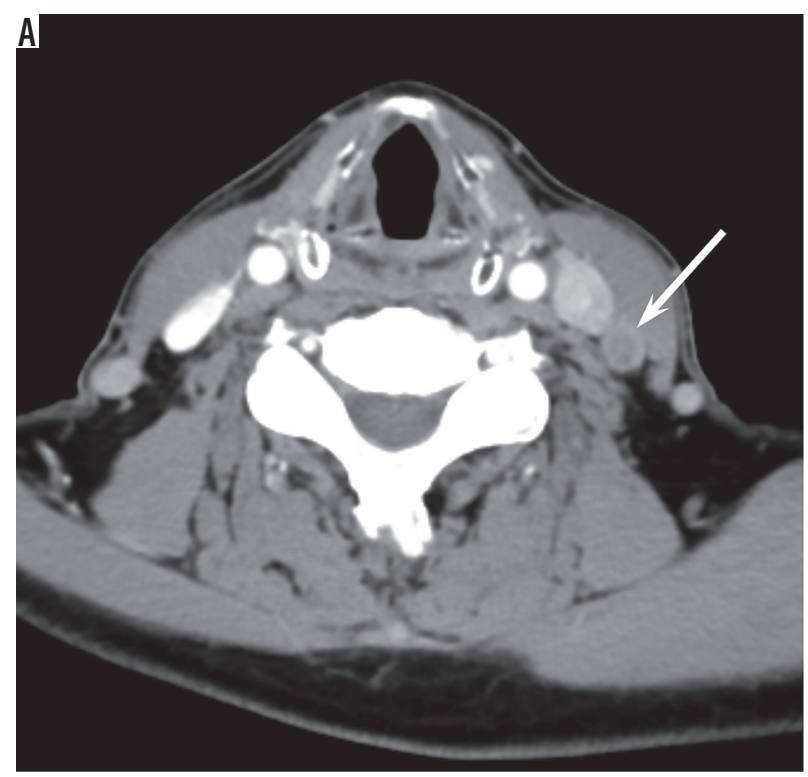

by Som et al. [17]. The surgeons who performed the neck dissections manually identified and localised the lymph nodes per neck level using the neck dissection specimens and recorded the maximum and minimum diameters of all nodal specimens. By comparing the location and size of the lymph nodes between nodal specimens and radiological assessment on CECT, it was possible to perform a topographical correlation for each lymph node. After the lymph node correlation, a pathologist reviewed all the nodal specimens and classified them into two categories: positive or negative for metastasis. Lymph nodes $<5 \mathrm{~mm}$ in maximum diameter on CECT were excluded from this study because they were more likely to be histologically diagnosed as negative for metastasis, and it was difficult to identify lymph nodes by pathological-radiological correlation.

\section{Statistical analysis}

All statistical analyses were performed using MedCalc 12.7.2 (MedCalc Software, Ostend, Belgium). The sensitivity, specificity, and accuracy for the detection of metastatic nodes were calculated, and McNemar's test was performed to compare the results between A-CECT and A\&C-CECT. Receiver operating characteristic curve analysis was used to determine the performance of the observers for each image set. Interobserver variability in the assessment of confidence level for the presence of metastatic nodes was analysed using kappa statistics.

\section{Results}

Among 120 dissected neck levels, a total of 238 histologically confirmed lymph nodes with a diameter $\geq 5 \mathrm{~mm}$

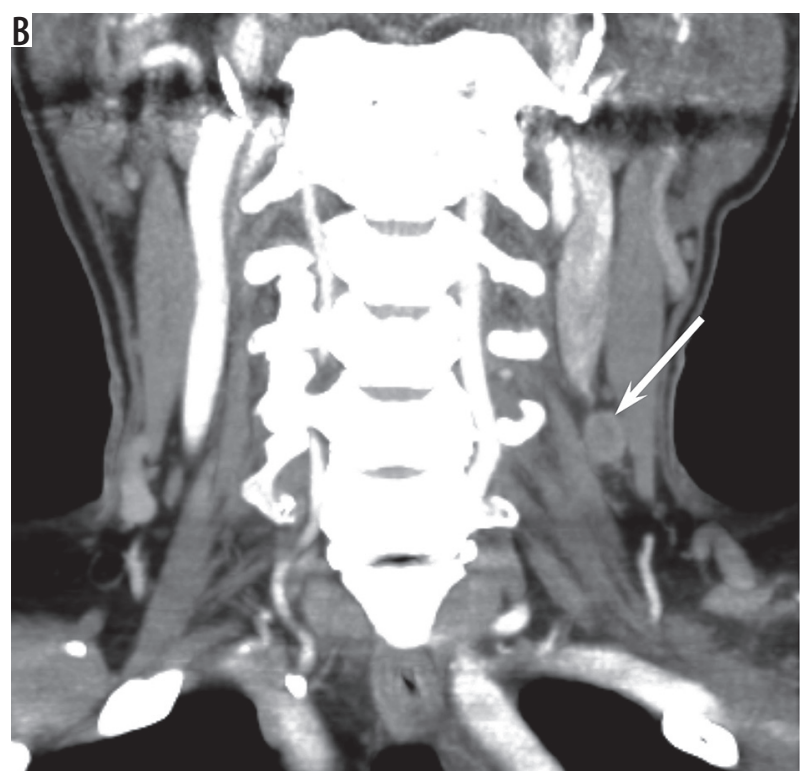

Figure 1. A 65-year-old man with tongue squamous cell carcinoma. A) Axial contrast-enhanced computed tomography image shows a spherical (round)shaped left level III lymph node (arrow) with $8 \mathrm{~mm}$ in maximum short-axis diameter. Central necrosis is clearly demonstrated within the lymph node. B) Coronal contrast-enhanced computed tomography image also shows a spherical (round)-shaped lymph node (arrow) with central necrosis. Metastasis is pathologically confirmed within the lymph node 

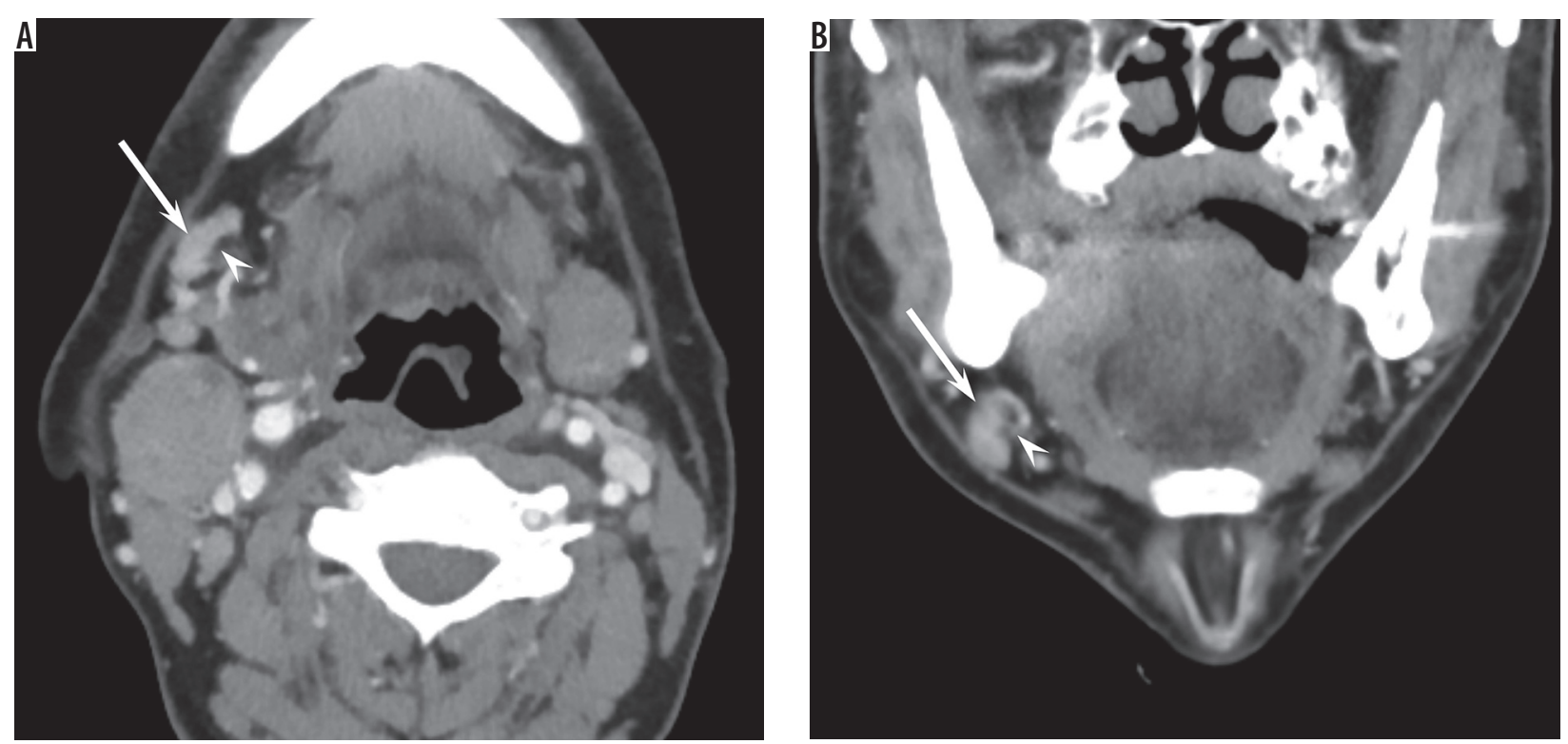

Figure 2. A 59-year-old man with oropharyngeal squamous cell carcinoma. A) Axial contrast-enhanced computed tomography image shows a flattened (oval)-shaped left level IB lymph node (arrow) with $6 \mathrm{~mm}$ in maximum short-axis diameter. Fatty hilum is clearly demonstrated (arrowhead). B) Coronal contrast-enhanced computed tomography image also shows a flattened (oval)-shaped lymph node (arrow) with fatty hilum (arrowhead). No evidence of metastasis is pathologically confirmed within the lymph node

Table 2. Sensitivity, specificity, accuracy, and AUC for the detection of metastatic lymph nodes for each observer

\begin{tabular}{|l|c|c|c|c|c|c|} 
& \multicolumn{3}{|c|}{ Observerer\#1 } & \multicolumn{3}{c|}{ Observerer \#2 } \\
\cline { 2 - 7 } & A-CECT & A\&C-CECT & $p$ value & A-CECT & A\&C-CECT & $p$ value \\
\hline Sensitivity & 73.2 & 75.3 & 0.727 & 69.1 & 69.1 & 1.000 \\
\hline Specificity & 92.2 & 97.2 & $0.039^{*}$ & 92.9 & 95.7 & 0.219 \\
\hline Accuracy & 84.5 & 88.2 & $0.049^{* *}$ & 83.2 & 85.3 & 0.302 \\
\hline AUC & 0.86 & 0.91 & $0.011^{\dagger}$ & 0.85 & 0.85 & 0.803 \\
\hline
\end{tabular}

AUC - area under the curve, A-CECT - axial contrast-enhanced computed tomography, A\&C-CECT - axial and coronal contrast-enhanced computed tomography

In sensitivity, specificity, and accuracy, data are percentages.

*Specificity of A\&C-CECT was greater than that of A-CECT $(p<0.05)$.

**Accuracy of A\&C-CECT was greater than that of A-CECT $(p<0.05)$.

${ }^{\dagger}$ AUC of A\&C-CECT was greater than that of A-CECT $(p<0.05)$.

(97 nodes with metastasis and 141 nodes without metastasis) were found (Figures 1 and 2). The distribution of the 238 lymph nodes was as follows: level I $(n=52)$, II $(n=103)$, III $(n=49)$, IV $(n=23), \mathrm{V}(n=5)$, and VI $(n=6)$.

The sensitivity, specificity, accuracy, and area under curve (AUC) of A-CECT and A\&C-CECT for the detection of metastatic lymph nodes are shown in Table 2. The sensitivity of A-CECT vs. A\&C-CECT was $73.2 \%$ vs. $75.3 \%$ for observer $\# 1$ ( $p=0.73)$ and $69.1 \%$ vs. $69.1 \%$ for observer \#2 ( $p=1.00)$, respectively. The specificity of A-CECT vs. A\&C-CECT was $92.2 \%$ vs. $97.2 \%$ for observer \#1 $(p<0.05)$ and $92.9 \%$ vs. $95.7 \%$ for observer \#2 $(p=0.22)$, respectively. The accuracy of A-CECT vs. A\&C-CECT was $84.5 \%$ vs. $88.2 \%$ for observer \#1 $(p<0.05)$ and $83.2 \%$ vs. $85.3 \%$ for observer $\# 2(p=0.30)$, respectively. The area under the curve (AUC) of A-CECT versus A\&C-CECT was 0.86 vs. 0.91 for observer \#1 $(p<0.05)$ and 0.85 vs. 0.85 for observer \#2 $(p=0.80)$, respectively.
For the two observers, the $\kappa$ values in the assessment of confidence level for the presence of metastatic nodes using A-CECT showed substantial agreement (0.73), and those using A\&C-CECT also showed substantial agreement (0.71).

\section{Discussion}

In the present study, the specificity, accuracy, and AUC significantly improved by adding C-CECT to A-CECT during the assessment by the expert radiologist in head and neck imaging. In other words, the expert radiologist could obtain useful morphological information from C-CECT for the diagnosis of metastatic nodes. In contrast, although there was no significant difference in the sensitivity, specificity, accuracy, and AUC during the assessment by the general radiologist, the specificity slightly increased by adding C-CECT to A-CECT. The increase in specificity might help avoid overdiagnosis and overtreatment. 

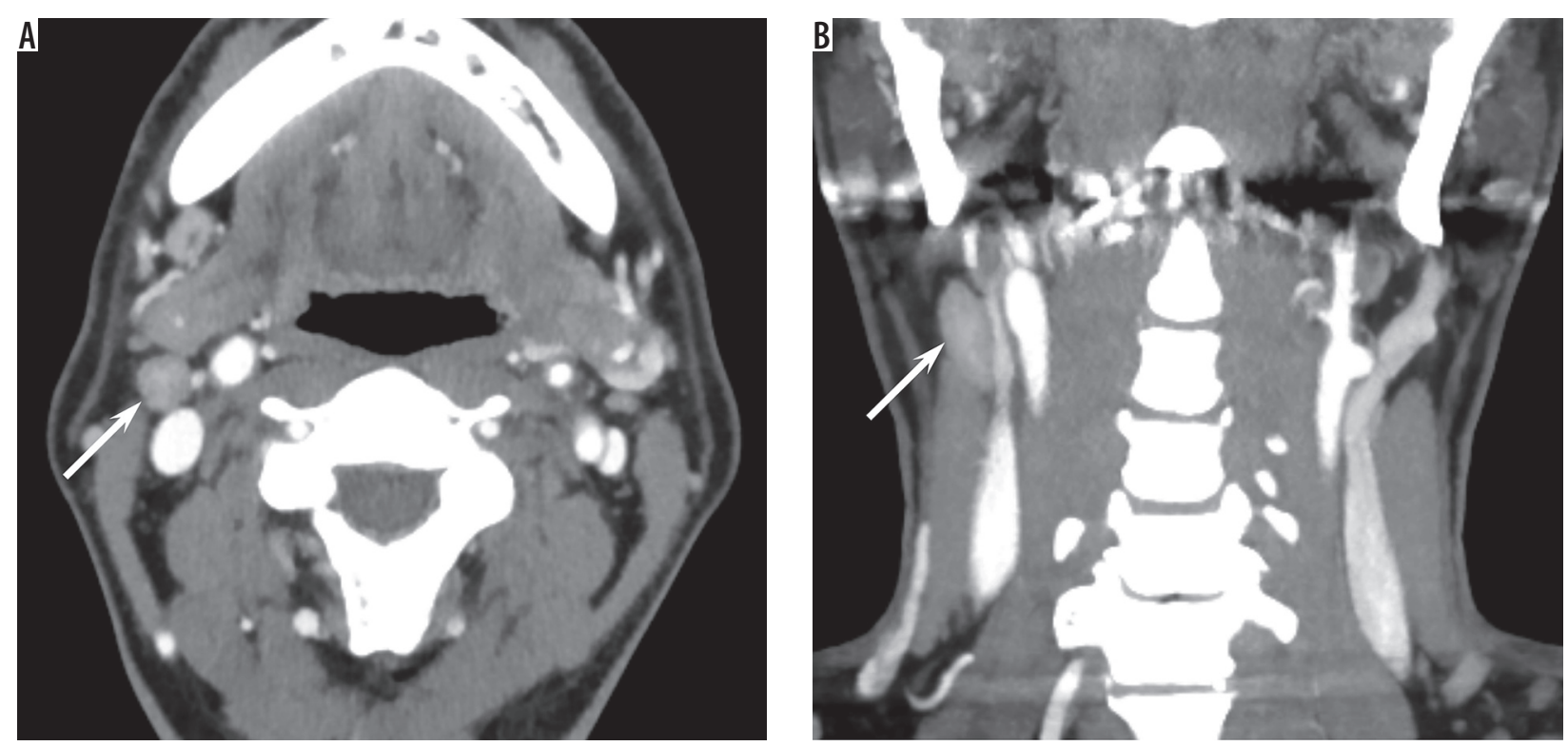

Figure 3. A 59-year-old man with gingival squamous cell carcinoma. A) Axial contrast-enhanced computed tomography image shows a spherical (round)shaped right level IIA lymph node (arrow) with $9 \mathrm{~mm}$ in maximum short-axis diameter. The reviewers judged a score of 3 (probably present) because of the spherical configuration. B) Coronal contrast-enhanced computed tomography image reveals a flattened (oval)-shaped lymph node (arrow), and the maximum diameter of this lymph node is $25 \mathrm{~mm}$. No evidence of metastasis is pathologically confirmed within the lymph node. An expert radiologist can accurately modify the judgement to a score of 2 (probably absent)
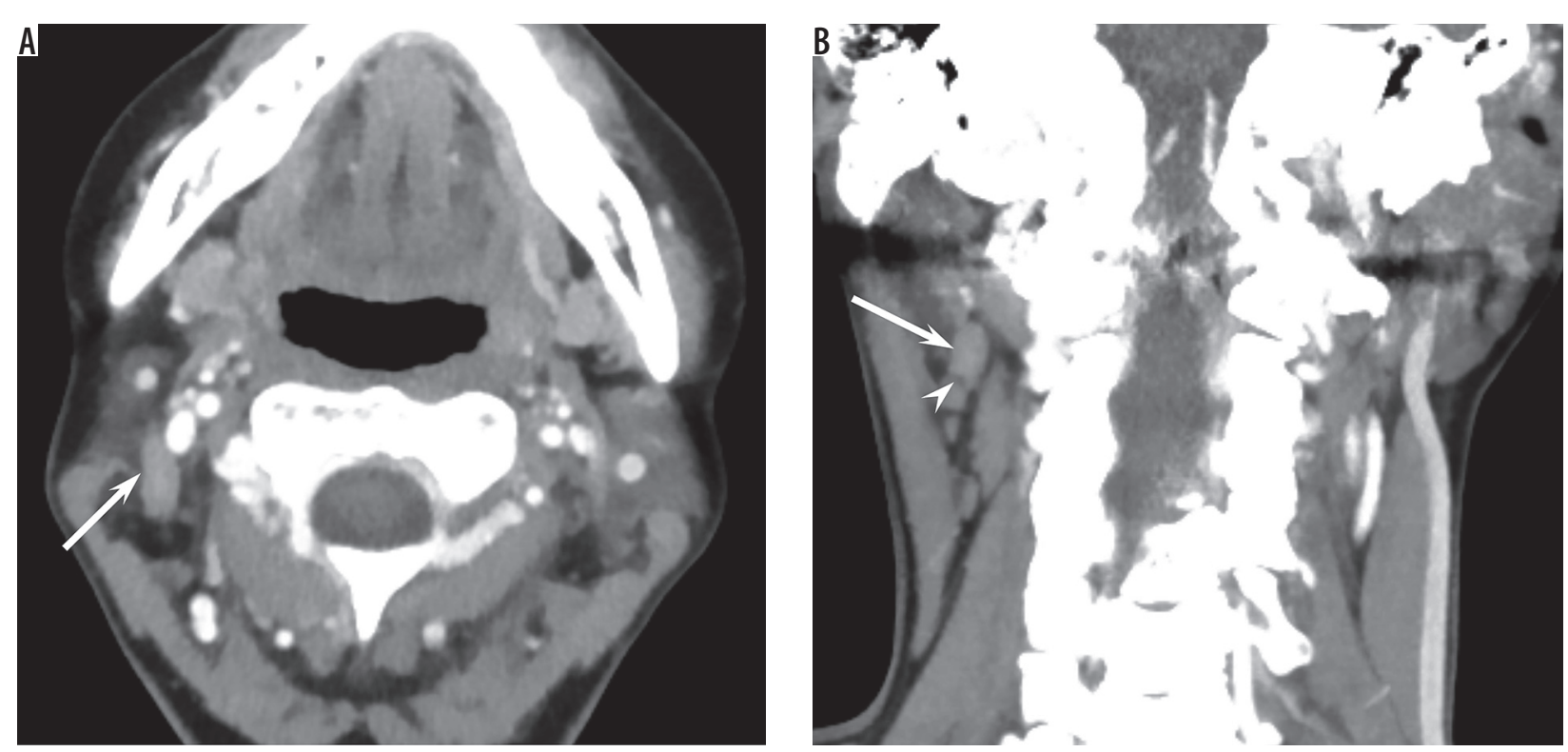

Figure 4. A 53-year-old woman with gingival squamous cell carcinoma. A) Axial contrast-enhanced computed tomography image shows a flattened (oval)-shaped right level IIA lymph node (arrow) with $9 \mathrm{~mm}$ in maximum short-axis diameter. The reviewers judged a score of 3 (probably present) because of the loss of fatty hilum. B) Coronal contrast-enhanced computed tomography image reveals a flattened (oval)-shaped lymph node (arrow) with the fatty hilum (arrowhead) at the lower end of lymph node. No evidence of metastasis is pathologically confirmed within the lymph node. An expert radiologist can accurately modify the judgement to a score of 2 (probably absent)

Of the 141 histologically proven non-metastatic lymph nodes, the expert radiologist judged eight nodes as false positive using A-CECT and as true negative using A\&C-CECT. In five of the eight lymph nodes, although spherical (round) shape on A-CECT led to a false positive diagnosis, flattened (oval) shape on C-CECT led to a true negative diagnosis (Figure 3). In the three remaining lymph nodes, although fatty hilum could not be detected on A-CECT, it could be detected on C-CECT (Figure 4).

The size of the lymph nodes is usually evaluated using both short- and long-axis diameters. Although the larger the size of the lymph node, the greater the risk of malignancy, benign reactive lymphadenitis or reactive hyperplasia of the lymph node can also result in nodes as large as malignant ones. For that reason, the short-axis 
diameter, which is measured perpendicular to the longest diameter of the lymph node, is recommended for the assessment of nodal metastasis [8]. Short-axis/long-axis (SL) ratio has also been widely used to differentiate reactive nodes from metastatic ones as a size criterion $[18,19]$. Specifically, benign lymph nodes tend to have an SL ratio of $<0.5$, whereas metastatic lymph nodes tend to have an SL ratio of $>0.5$ on both CT [18] and ultrasonography [19]. Although this size criterion is relatively simple and easy to follow, evaluation using axial CT images alone may sometimes fail to measure the true short- and long-axis diameters. If elliptical lymph nodes occur along the axis of the body, they might be regarded as malignant due to the spherical (round) configuration assessed by axial CT images alone (Figure 3). Thus, to measure the true short- and long-axis diameters, it is necessary to evaluate coronal or sagittal reconstruction CT images in addition to axial CT images.

The presence of fatty hilum is also one of the morphological features of benign lymph nodes, whereas its loss is an ancillary feature of malignancy $[11,19,20]$. Although the diagnostic criterion of the presence or absence of fatty hilum tends to have higher specificity, the sensitivity tends to be relatively low [11]. In some cases in our study, the detection of fatty hilum was improved by adding the assessment of C-CECT (Figure 4). The addition of C-CECT may also contribute to the prevention of misdiagnosis regarding the partial-volume effect of fatty hilum as central necrosis. However, if the intranodal cancer nest is small, fatty hilum may also be found in metastatic nodes [21].
In other words, although the addition of C-CECT may improve the diagnostic ability, it is still difficult to identify micrometastases within lymph nodes.

The present study has several limitations. First, the sample size was small because the study was conducted at a single institution. Second, due to the retrospective nature of this study, two different CT scanners were used. However, we believe that the results would not have differed considerably if we had obtained images using the same CT scanner. Third, in the present study, lymph nodes < $5 \mathrm{~mm}$ in maximum diameter were excluded because of the low probability of nodal metastasis and the difficulty in identification by pathological-radiological correlation. However, micrometastases might be present within these small lymph nodes.

\section{Conclusions}

Proper configuration recognition of cervical nodes using additional coronal reconstruction images improved the specificity, accuracy, and AUC for the detection of metastatic nodes in patients with HNSCC. The increase in specificity facilitated the accurate exclusion of nodal metastasis and might help avoid overdiagnosis and overtreatment.

\section{Conflict of interest}

The authors report no conflict of interest.

\section{References}

1. Leemans CR, Tiwari R, Nauta JJ, et al. Recurrence at the primary site in head and neck cancer and the significance of neck lymph node metastases as a prognostic factor. Cancer 1994; 73: 187-190.

2. Clark J, Li W, Smith G, et al. Outcome of treatment for advanced cervical metastatic squamous cell carcinoma. Head Neck 2005; 27 $87-94$.

3. Jan JC, Hsu WH, Liu SA, et al. Prognostic factors in patients with buccal squamous cell carcinoma: 10-year experience. J Oral Maxillofac Surg 2011; 69: 396-404.

4. Kokemueller H, Rana M, Rublack J, et al. The Hannover experience: surgical treatment of tongue cancer - a clinical retrospective evaluation over a 30 years period. Head Neck Oncol 2011; 3: 27.

5. Scherpelz KP, Wong AC, Lingen MW, et al. Histological features and prognostic significance of treatment effect in lymph node metastasis in head and neck squamous cell carcinoma. Histopathology 2019; 74: 321-331.

6. Chu HR, Kim JH, Yoon DY, et al. Additional diagnostic value of (18)F-FDG PET-CT in detecting retropharyngeal nodal metastases. Otolaryngol Head Neck Surg 2009; 141: 633-638.

7. Kato H, Kanematsu M, Watanabe H, et al. Metastatic retropharyngeal lymph nodes: comparison of CT and MR imaging for diagnostic accuracy. Eur J Radiol 2014; 83: 1157-1162.
8. van den Brekel MW, Stel HV, Castelijns JA, et al. Cervical lymph node metastasis: assessment of radiologic criteria. Radiology 1990; 177: 379-384.

9. Curtin HD, Ishwaran H, Mancuso AA, et al. Comparison of CT and MR imaging in staging of neck metastases. Radiology 1998; 207: 123-130.

10. Sakai O, Curtin HD, Romo LV, et al. Lymph node pathology. Benign proliferative, lymphoma, and metastatic disease. Radiol Clin North Am 2000; 38: 979-998.

11. Liu Z, Zeng W, Liu C, et al. Diagnostic accuracy of ultrasonographic features for lymph node metastasis in papillary thyroid microcarcinoma: a single-center retrospective study. World J Surg Oncol 2017; 15: 32.

12. Wu LM, Xu JR, Liu MJ, et al. Value of magnetic resonance imaging for nodal staging in patients with head and neck squamous cell carcinoma: a meta-analysis. Acad Radiol 2012; 19: 331-340.

13. Sun R, Tang X, Yang Y, et al. (18)FDG-PET/CT for the detection of regional nodal metastasis in patients with head and neck cancer: a meta-analysis. Oral Oncol 2015; 51: 314-320.

14. Bartlett ES, Walters TD, Yu E. Can axial-based nodal size criteria be used in other imaging planes to accurately determine "enlarged" head and neck lymph nodes? ISRN Otolaryngol 2013; 2013: 232968. 
15. Chen C, Zhang M, Xu Y, et al. Unidimensional measurement may evaluate target lymph nodal response after induction chemotherapy for nasopharyngeal carcinoma. Medicine (Baltimore) 2016; 95: e2667.

16. Schreyer AG, Scheibl K, Zorger N, et al. Detection rate and efficiency of lymph node assessment with axial and coronal image reading based on 16 row multislice CT of the neck. Rofo 2005; 177: 1430 1435.

17. Som PM, Curtin HD, Mancuso AA. Imaging-based nodal classification for evaluation of neck metastatic adenopathy. AJR Am J Roentgenol 2000; 174: 837-844.
18. Steinkamp HJ, Hosten N, Richter C, et al. Enlarged cervical lymph nodes at helical CT. Radiology 1994; 191: 795-798.

19. Vj R, A SA, Mufeed A, et al. Characterization of cervicofacial lymphnodes - a clinical and ultrasonographic study. J Clin Diagn Res 2014; 8: ZC25-28.

20. Elson M, Rothman M, Ord RA. False-positive computed tomography scan mimicking metastasis due to fatty hilum in a cervical lymph node. J Oral Maxillofac Surg 1994; 52: 1334-1336.

21. Vassallo P, Edel G, Roos N, et al. In-vitro high-resolution ultrasonography of benign and malignant lymph nodes. A sonographic-pathologic correlation. Invest Radiol 1993; 28: 698-705. 